\title{
Low-complexity DOA estimation from short data snapshots for ULA systems using the annihilating filter technique
}

\author{
Faouzi Bellili* ${ }^{*}$, Souheib Ben Amor ${ }^{1}$, Sofiène Affes ${ }^{1}$ and Ali Ghrayeb ${ }^{2}$
}

\begin{abstract}
This paper addresses the problem of DOA estimation using uniform linear array (ULA) antenna configurations. We propose a new low-cost method of multiple DOA estimation from very short data snapshots. The new estimator is based on the annihilating filter (AF) technique. It is non-data-aided (NDA) and does not impinge therefore on the whole throughput of the system. The noise components are assumed temporally and spatially white across the receiving antenna elements. The transmitted signals are also temporally and spatially white across the transmitting sources. The new method is compared in performance to the Cramér-Rao lower bound (CRLB), the root-MUSIC algorithm, the deterministic maximum likelihood estimator and another Bayesian method developed precisely for the single snapshot case. Simulations show that the new estimator performs well over a wide SNR range. Prominently, the main advantage of the new AF-based method is that it succeeds in accurately estimating the DOAs from short data snapshots and even from a single snapshot outperforming by far the state-of-the-art techniques both in DOA estimation accuracy and computational cost.
\end{abstract}

Keywords: DOA estimation, Root-MUSIC, Annihilating filter, Array signal processing, NDA estimation

\section{Introduction}

In recent years, there has been a surge of interest in array signal processing applications in both military and civil domains $[1,2]$. The concept of direction of arrival (DOA) estimation find its use in applications related to radar or sonar systems. In addition, in modern mobile communication systems, for example, based only on the data received at the antenna array, estimating the DOAs of the desired users and those of the interference signals allows their extraction and cancellation, respectively, by beamforming technologies $[3,4]$ in order to improve the wireless systems' performance.

Roughly speaking, depending on the a priori knowledge of the transmitted signals, DOA estimators can be categorized as data-aided (DA) or non-data-aided (NDA). In plain English, DA approaches base the estimation process on a priori perfectly known symbols. Unfortunately, although being simple and accurate, these approaches may

*Correspondence: bellili@emt.inrs.ca

${ }^{1}$ INRS-EMT, 800, de la Gauchetière Ouest, Suite 6900, H5A 1 K6 Montréal, QC, Canada

Full list of author information is available at the end of the article suffer from the major drawback of limiting the whole throughput of the system by periodically sending a reference (known) signal [5]. It should be mentioned here that superimposed pilots do not affect the throughput but increase the complexity of the channel estimation process. Hence, the ever increasing demand for channel bandwidth spurred the more practically oriented minds to develop new estimation techniques that rely on the received data samples only and which are therefore commonly known as NDA techniques. NDA estimators themselves are referred to as deterministic or stochastic if the unknown transmitted signal is assumed deterministic or completely random, respectively. So far, from maximum likelihood-based to subspace-based methods, many NDA DOA estimators have been proposed and extensively studied in the literature [6-8]. The NDA maximum likelihood approaches are undoubtedly the most accurate, but unfortunately, they are often computationally very expensive. To circumvent this challenging problem, covariance-based estimators are often a trend-in NDA estimation schemes-to alleviate this burden of computational cost. Fortunately, usually, 
they also provide sufficiently accurate DOA estimates, especially in the presence of sufficiently large number of received samples. But in situations of short data snapshots, they may not be reliable and one would be obliged to trade low complexity for more accurate estimation by simply applying the maximum likelihood approaches. Yet, the maximum likelihood estimators are analytically intractable in the NDA case especially in the presence of random transmitted symbols/signals. Therefore, they are often tackled numerically via multidimensional grid search approaches. Their accuracy/resolution is therefore dictated by the discretization step of the grid. A very dense discretization (small step) is able to provide very accurate estimates even at low operational SNRs, but the complexity of the underlying ML algorithm would be extremely high and even prohibitive since its complexity grows exponentially with the number of the parameters to be estimated. Another alternative is to solve the ML criterion using pilot/reference symbols/signals only where a closed-form solution may be feasible. Unfortunately, this approach is not able to provide in-service estimates as the receiver is compelled to wait for the next pilot signals in order to update the estimates.

Motivated by these facts, we develop in this paper a new covariance-based DOA estimation method for ULA configurations which succeeds in estimating the DOA from very short data records. It is based on the annihilating filter technique: finding the roots of an annihilating filter (AF) which are directly related to the unknown DOAs. It should be noted that the AF technique has been well known for a very long time in the mature field of spectral estimation. About a decade ago, it was also used to successfully develop the so-called finite-rate-of-innovation (FRI) sampling method [9] where it led to signal sampling and reconstruction paradigms at the minimal possible rate (far below the traditional Nyquist rate). In this contribution, we apply for the first time the AF approach to DOA estimation for ULA configurations and, therefore, we will henceforth refer to our new technique as the AF-based method. The coefficients of the corresponding AF are calculated by the singular value decomposition (SVD) of a matrix whose elements are built from second-order cross moments across the receiving antenna elements of the received samples. Interestingly, this matrix is of reduced dimensions thereby yielding a very low computational load of the SVD decomposition.

We propose two different versions of the new AF-based solution ${ }^{1}$ depending on the SNR threshold. The first one, referred to as "version I", is more advantageous at high SNR levels. It exploits each consecutive $2 K+1$ correlation coefficients along the columns and rows of the covariance matrix ( $K$ being the number of sources). The second one, referred to as "version II", exploits the Toepltiz structure of the covariance matrix in order to enhance the estimation performance at low SNR levels. In both versions, the obtained DOA estimates are then used to find the unknown sources' powers along with the noise variance.

In the multiple snapshot case, both versions of the proposed AF-based technique are compared in accuracy performance to the Cramér-Rao lower bound (CRLB) [10] and to the root-MUSIC algorithm-a popular and powerful technique of DOA estimation for ULA systemswhich is also based on polynomial rooting [11]. In the single-snapshot scenario, however, it is compared to another Bayesian method that was designed precisely for the challenging single-snapshot case [12] as well as the deterministic ML (DML) estimator. We mention here that a more recent iterative technique that handles the singlesnapshot case has also been proposed in [13]. Unfortunately, in its NDA version, it relies on the prior availability of an initial guess about all the unknown DOAs whose accuracy affects the overall performance of the method. Therefore, for the sake of fairness, this technique is not considered since none of the considered techniques (including our AF-based estimator itself) requires an initial guess about the DOAs. Even more, it has been recently recognized in a comparative study of various DOA estimators [14] that DML is indeed the most attractive one if the DOAs are to be estimated from a single snapshot. It will be shown by Monte-Carlo simulations that the new AF-based method is able to accurately estimate the DOAs from short data snapshots and even from a single-shot measurement. Furthermore, it outperforms the classical Bayesian and DML estimators over a wide SNR range with a slight performance advantage for the latter in the low SNR region but at the cost of an extremely high computational load.

We organize the rest of this paper as follows. In Section 2, we introduce the system model that will be used throughout this article. Then in Section 3, we develop our new AF-based DOA estimation technique. In Section 4, we exploit these new AF-based DOA estimates to develop new estimates for the channel powers. In Section 5, we assess the performance of the new estimators. Finally, we draw out some concluding remarks in Section 6.

We mention beforehand that some of the common notations will be used throughout this paper. Vectors and matrices are represented by lower- and upper-case bold fonts, respectively. Moreover, $\{.\}^{H}$ and $\{.\}^{T}$ denote the Hermitian (i.e. transpose conjugate) and transpose of any vector or matrix, respectively. The operators $\{.\}^{*}$ and $|$. return the conjugate and amplitude of any complex number, respectively, and $j$ is the pure complex number that verifies $j^{2}=-1$. Moreover, $N_{a}$ refers to the number of antenna elements in a uniform linear array (ULA). The statistical expectation is denoted as $E\{$.$\} , and the notation$ $\triangleq$ is used for definitions. 


\section{System model}

We consider a uniform linear array (ULA) of $N_{a}$ antenna elements immersed in a homogeneous media in the far field of $K$ point sources that are transmitting multiple planar waves. We assume that the transmitted signals are temporally white and uncorrelated between the radiating sources. Assuming perfect frequency synchronization, the received signal on the $\left\{i^{t h}\right\}_{i=1}^{N_{a}}$ antenna element, at the output of the matched filter, can be modelled as a complex signal as follows:

$$
y_{i}(n)=\sum_{k=1}^{K} h_{k} e^{j(i-1) \pi \sin \left(\theta_{k}\right)} a_{k}(n)+w_{i}(n),
$$

where at time index $n, a_{k}(n)^{2}$ is the signal (or symbol) transmitted by the $k^{\text {th }}$ source and $w_{i}(n)$ is the noise component on the $i^{\text {th }}$ antenna branch that is modelled by a zero-mean complex Gaussian random variable with independent real and imaginary parts, each of variance $\sigma^{2}$. The complex channel coefficients corresponding to the $K$ sources are assumed to be unknown, and they are denoted by $\left\{h_{k}=\left|h_{k}\right| e^{j \phi_{k}}\right\}_{k=1}^{K}$ where $\phi_{k}$ stands for any possible channel distortion phase. Moreover, $\left\{\theta_{k}\right\}_{k=1}^{K}$ are the unknown DOAs (to be estimated) of the planar waves impinging from the $K$ sources.

Note here that the receiving antenna elements are supposed to be spaced by half the wavelength, i.e. $d=$ $\lambda / 2$ where $d$ is the distance between two consecutive antenna branches and $\lambda$ is the carrier wavelength of the signal. Note also that although the vector/matrix representation of the received signals is more compact and widely adopted in the open literature, we settle here on the scalar form of the received signals (i.e. the elementary received signals on each antenna element). We believe that this representation allows for an easy grasp of the theoretical foundations of the new estimator since it isas will be seen later-based on the explicit expression for each cross-covariance between the elementary received signals.

We assume hereafter that at each time instant $n$ the transmitted signals, $\boldsymbol{a}(n)=\left[a_{1}(n), a_{2}(n), \cdots, a_{K}(n)\right]^{T}$ and the noise components $\boldsymbol{w}(n)=\left[w_{1}(n), \cdots, w_{N_{a}}(n)\right]^{T}$ are each uncorrelated element-wise, i.e.

$$
E\left\{\boldsymbol{w}(n)^{H} \boldsymbol{w}(n)\right\}=2 \sigma^{2} \boldsymbol{I}_{N_{a}} \text { and } E\left\{\boldsymbol{a}(n)^{H} \boldsymbol{a}(n)\right\}=\boldsymbol{I}_{K},
$$

where in the last equality, we assume, without loss of generality, that the energy of the transmitted signals are normalized to one, i.e. $E\left\{\left|a_{k}(n)\right|^{2}\right\}=1$. In fact, the transmitted powers, $P_{k}=\mathrm{E}\left\{\left|a_{k}(n)\right|^{2}\right\}$, can always be incorporated in the channel coefficients after being scaled by the factor $\sqrt{P_{k}}$. Finally, the symbols, $\left\{a_{k}(n)\right\}_{n=1}^{N}$, transmitted by source $k$ over the observation time window are assumed mutually independent. Then, we define the true SNR of the $k^{\text {th }}$ source as follows:

$$
\rho_{k} \triangleq \frac{E\left\{\left|h_{k}\right|^{2}\left|a_{k}(n)\right|^{2}\right\}}{2 \sigma^{2}}=\frac{\left|h_{k}\right|^{2}}{2 \sigma^{2}} .
$$

\section{Formulation of the new AF-based DOA estimator}

In few words, we mention that the new AF-based estimator relies on a special property that is inherent to appropriately selected sequences of second-order cross-moments of the received signals. Therefore, we will simply begin by deriving the explicit expression of the elementary crosscovariances between the different antenna branches. To that end, and by assuming a perfect knowledge of the number of signals $K$, we gather for more convenience all the unknown DOAs in one single parameter vector $\boldsymbol{\theta}=$ $\left[\theta_{1}, \theta_{2}, \cdots, \theta_{K}\right]^{T}$. Then, the cross-covariances between the received signals from any pair $(i, l)$ of the receiving antenna array can be defined as:

$$
\Sigma_{y}(i, l) \triangleq E\left\{y_{i}(n) y_{l}^{*}(n)\right\}, i, l=1,2, \cdots, N_{a} .
$$

Recall the fact that the transmitted signals and the noise components are spatially and temporally white; hence, $M_{\theta}(i, l)$ reduces simply to:

$$
\Sigma_{y}(i, l)= \begin{cases}\sum_{k=1}^{K}\left|h_{k}\right|^{2}+2 \sigma^{2} & \text { for } i=l, \\ \sum_{k=1}^{K}\left|h_{k}\right|^{2} e^{j \pi(i-l) \sin \left(\theta_{k}\right)} & \text { for } i \neq l .\end{cases}
$$

In practice, all the cross-covariances, $\left\{\Sigma_{y}(i, l)\right\}_{i, l=1}^{N_{a}}$, can be easily computed together using the vector/matrix representation of the received signals. Indeed, denoting by

$$
\boldsymbol{y}(n)=\left[y_{1}(n), y_{2}(n), \cdots, y_{N_{a}}(n)\right]^{T},
$$

the received vector at time instant $n, \Sigma_{y}(i, l)$, is nothing but the $(i, l)^{\text {th }}$ entry of the covariance matrix, $\boldsymbol{\Sigma}_{\boldsymbol{y}}=$ $\mathrm{E}\left\{\boldsymbol{y}(n) \boldsymbol{y}^{H}(n)\right\}$. The latter matrix is Toeplitz structured ${ }^{3}$ due to the use of an ULA antenna and can be estimated by a simple sample mean as follows:

$$
\widehat{\boldsymbol{\Sigma}}_{\boldsymbol{y}}=\frac{1}{N} \sum_{n=1}^{N} \boldsymbol{y}(n) \boldsymbol{y}^{H}(n) .
$$

We mention here that since $\boldsymbol{\Sigma}_{\boldsymbol{y}}$ (and $\widehat{\boldsymbol{\Sigma}}_{\boldsymbol{y}}$ ) is a Hermitian matrix (i.e. $\boldsymbol{\Sigma}_{\boldsymbol{y}}=\boldsymbol{\Sigma}_{\boldsymbol{y}}^{H}$ ), then the strictly lower triangular matrix obtained from $\boldsymbol{\Sigma}_{\boldsymbol{y}}$ contains all the information about the DOAs that would be extracted from the entire matrix. Indeed, the diagonal elements do not depend on the unknown DOAs, although they can be eventually used to estimate the noise variance after estimating the channel coefficients from the off-diagonal entries as detailed later. Consequently, from now on, the counters $i$ and $l$ will always verify ${ }^{4} i>l$. Then, using the notation $u_{k}=$ $e^{j \pi \sin \left(\theta_{k}\right)}$, we define the $N_{a}-1$ sequences-indexed by the counter $l-\left\{r_{\boldsymbol{\theta}}^{(l)}[m]\right\}_{m=1}^{N_{a}-l}$, each of which containing the $N_{a}-l$ elements of the $\left\{l^{t h}\right\}_{l=1}^{N_{a}-1}$ column that are lying strictly below the main diagonal as follows: 


$$
r_{\boldsymbol{\theta}}^{(l)}[m]=\Sigma_{y}(l+m, l), m=1,2, \cdots, N_{a}-l,
$$

which is simply given by

$$
r_{\boldsymbol{\theta}}^{(l)}[m]=\sum_{k=1}^{K}\left|h_{k}\right|^{2} u_{k}^{m}, m=1,2, \cdots, N_{a}-l .
$$

These terms can be stacked together in a $\left(N_{a}-l\right)$ dimensional vector $\boldsymbol{r}_{\boldsymbol{\theta}}^{(l)}$-that will be used subsequentlyas follows ${ }^{5}$ :

$$
\boldsymbol{r}_{\boldsymbol{\theta}}^{(l)}=\left[r_{\theta}^{(l)}[1], r_{\theta}^{(l)}[2], \cdots, r_{\theta}^{[l]}\left[N_{a}-l\right]\right]^{T} .
$$

Now, we see from (9) that each sequence $\left\{r_{\boldsymbol{\theta}}^{(l)}[m]\right\}_{m=1}^{N_{a}-l}$ is simply a weighted sum of exponentials. This interesting property is very useful-when combined with the annihilating filter technique-and is actually the main idea behind this work as will be soon explained.

Generally speaking, a filter $g[\mathrm{~m}]$ is called an annihilating filter of a signal or more generally a discrete sequence $\{s[m]\}_{m}$ when

$$
(g * s)[m]=0, \quad \forall m \in \mathbb{Z},
$$

where $*$ stands for the discrete convolution. Usually, the filtering operation is applied to signals. But in this paper, we filter a sequence of cross-covariances by interpreting them as received samples. Therefore, the crosscovariances sequence $\left\{r_{\boldsymbol{\theta}}^{(l)}[m]\right\}_{m=1}^{N_{a}-l}$ will play the role (interpreted as) of the signal sequence $s[m]$ in (11). Indeed, as shown subsequently, for such special sequences (linear combinations of exponentials), the roots of the corresponding annihilating filters are exactly the involved elementary exponentials. More formally, consider the following filter:

$$
\begin{aligned}
g(z) & \triangleq \prod_{k=1}^{K}\left(1-u_{k} z^{-1}\right), \\
& =\sum_{n=0}^{K} g[n] z^{-n} .
\end{aligned}
$$

Then, we have:

$$
\begin{aligned}
\left(g * r_{\boldsymbol{\theta}}^{(l)}\right)[m] & =\sum_{n=0}^{K} g[n] r_{\boldsymbol{\theta}}^{(l)}[m-n], \\
& =\sum_{n=0}^{K} \sum_{m=1}^{K}\left|h_{k}\right|^{2} g[n] u_{k}^{m-n} \\
& =\sum_{m=1}^{K}\left|h_{k}\right|^{2} \underbrace{\left(\sum_{n=0}^{K} g[n] u_{k}^{-n}\right)}_{A\left(u_{k}\right)=0} u_{k}^{m}, \\
& =0 .
\end{aligned}
$$

Therefore, $g[n]$-as constructed in (12) - is indeed an annihilating filter for the sequence $\left\{r_{\theta}^{(l)}[m]\right\}_{m=1}^{N_{a}-1}$. Then, if one is able to find the coefficients $\{g[n]\}_{n=0}^{K}$, the roots of the corresponding polynomial $g(z)$ in (12) would be easily computed and then the DOAs can be easily estimated from the arguments of the obtained roots. To that end, we gather the desired coefficients, $\{g[n]\}_{n=0}^{K}$, in a single unknown vector $\boldsymbol{g}=[g[0], g[2], \cdots, g[K]]^{T}$ and describe below an easy SVD procedure that enables finding $g$.

First notice that the unknown filter coefficients $\{g[n]\}_{n=0}^{K}$ in $g(z)=\sum_{n=0}^{K} g[n] z^{-n}$ must be such that (11) is satisfied for all $m \in \mathbb{Z}$ and in particular for $m>n$ :

$$
\sum_{n=0}^{K} g[n] r_{\boldsymbol{\theta}}^{(l)}[m-n]=0 \forall m>n .
$$

Then, using the elements, $r_{\boldsymbol{\theta}}^{(l)}[m-n]$, extracted from the $l^{\text {th }}$ column of the covariance matrix, we estimate (as described later) from (14) the $K+1$ unknown filter coefficients. In this way, it is clear that one needs $K+1$ independent equations - obtained by changing $m$-in order to obtain at least one estimate, $\hat{\boldsymbol{g}}^{(l)}$, of the desired vector $\boldsymbol{g}$. Therefore, if a column $l$ is to be useful, the corresponding vector $\boldsymbol{r}_{\boldsymbol{\theta}}^{(l)}$ should contain at least $2 K+1$ elements. Recall from (10) that the size of $\boldsymbol{r}_{\boldsymbol{\theta}}^{(l)}$ is $N_{a}-l$, which results in $N_{a}-l \geq 2 K+1$. Therefore, $l$ must verify

$$
1 \leq l \leq N_{a}-2 K-1
$$

meaning that only the first $N_{a}-2 K-1$ columns of the covariance matrix contain a sufficient number of crosscovariances that enable having at least one estimate, $\hat{\boldsymbol{g}}^{(l)}$ of $\boldsymbol{g}$, per-column (or per-vector $\boldsymbol{r}_{\boldsymbol{\theta}}^{(l)}$ ). Observe also from (15) that it is necessary to have at least $N_{a} \geq 2 K+2$ receiving antenna elements for $K$ unknown sources. Thus, our estimator needs more than twice the number of antennas as the number of sources. Moreover, in addition to the trivial initial estimate, $\widehat{\boldsymbol{g}}_{0}^{(l)}$, that is obtained using the first necessary $2 K+1$ cross-covariances in $\boldsymbol{r}_{\boldsymbol{\theta}}^{(l)}$, we can actually obtain $P_{l}$ additional estimates $\left\{\hat{\boldsymbol{g}}_{p}^{(l)}\right\}_{p=1}^{P_{l}}$ for the unknown $\boldsymbol{g}$ from each candidate column $l$. Here, $P_{l}=N_{a}-l-2 K-1$ is the number of samples exceeding these necessary first $2 K+1$ cross-covariances. This means that we obtain $P_{l}+1=N_{a}-2 K-l$ estimates for $\boldsymbol{g}$ from each eligible column $l$. In fact, for a given $l$, the $\left\{l^{\text {th }}\right\}_{l \leq N_{a}-2 K-1}$ vector $\boldsymbol{r}_{\boldsymbol{\theta}}^{(l)}$ contains $2 K+1+P_{l}$ cross-covariances. Then, for each $p=0,1,2, \cdots, P_{l}$, consider $2 K+1$ consecutive samples of these second-order moments, $\left\{r_{\boldsymbol{\theta}}^{(l)}\left[m_{p}+r\right]\right\}_{r=-K}^{K}$, that are centred around $m_{p}=K+1+p$. Now, replacing $m$ by $m_{p}+r$, the system in (14) yields 


$$
\sum_{n=0}^{K} g[n] r_{\theta}^{(l)}\left[m_{p}+r-n\right]=0, r=-K,-K+1, \cdots, K-1, K
$$

Therefore, for each value of $p$ (or equivalently $m_{p}$ ), we have $K+1$ independent equations which can be more conveniently written in the matrix/vector form as follows:

$$
\boldsymbol{S}_{p}^{(l)}(\boldsymbol{\theta}) \boldsymbol{g}=\mathbf{0}
$$

where

$$
\begin{aligned}
& \boldsymbol{S}_{p}^{(l)}(\boldsymbol{\theta})= \\
& \left(\begin{array}{cccc}
r_{\boldsymbol{\theta}}^{(l)}[K+p+1] & r_{\boldsymbol{\theta}}^{(l)}[K+p] & \cdots & r_{\boldsymbol{\theta}}^{(l)}[p+1] \\
r_{\boldsymbol{\theta}}^{(l)}[K+p+2] & r_{\boldsymbol{\theta}}^{(l)}[K+p+1] & \cdots & r_{\boldsymbol{\theta}}^{(l)}[p+2] \\
\vdots & \vdots & \ddots & \vdots \\
r_{\boldsymbol{\theta}}^{(l)}[2 K+p+1] & r_{\boldsymbol{\theta}}^{(l)}[2 K+p] & \cdots & r_{\boldsymbol{\theta}}^{(l)}[K+1+p]
\end{array}\right) .
\end{aligned}
$$

Note that for each $l$, we have $P_{l}+1=N_{a}-l-2 K$ possible linear systems (by varying $p$ ) that provide $P_{l}+1$ estimates for the same vector $g$-involved in these systems-as previously stated.

In practice, the system in (17) can be solved via a singular value decomposition (SVD) where the $(K+1 \times K+1)$ matrix $\boldsymbol{S}_{p}^{(l)}(\boldsymbol{\theta})$ is decomposed into:

$$
\boldsymbol{S}_{p}^{(l)}(\boldsymbol{\theta})=\boldsymbol{U}_{p}^{(l)}(\boldsymbol{\theta}) \boldsymbol{D}_{p}^{(l)}(\boldsymbol{\theta}) \boldsymbol{V}_{p}^{(l)}(\boldsymbol{\theta})^{H}
$$

Then, for each $l=1,2, \cdots, N_{a}-2 K-1$ and $p=$ $0,1, \cdots,\left(N_{a}-2 K-1\right)-l$, we obtain an estimate, $\hat{\boldsymbol{g}}_{p}^{(l)}$, for $g$ as follows:

$$
\hat{\boldsymbol{g}}_{p}^{(l)}=\boldsymbol{V}_{p}^{(l)}(\boldsymbol{\theta}) \boldsymbol{e}_{K+1},
$$

where $\boldsymbol{e}_{K+1}$ is a vector with 1 at position $K+1$ and 0 elsewhere. Solving for the $K$ roots of $\hat{\boldsymbol{g}}_{p}^{(l)}$, we obtain a set of estimates for $\left\{u_{k}=e^{j \pi \sin \left(\theta_{k}\right)}\right\}_{k=1}^{K}$. We denote these estimates as $\left\{\hat{u}_{k}^{(l, p)}\right\}_{k=1}^{K}$ from which a set of estimates for the unknown DOAs are obtained for each $l$ and $p$ as follows:

$$
\hat{\theta}_{k}^{(l, p)}=\frac{1}{\pi} \arcsin \left(\angle \hat{u}_{k}^{(l, p)}\right), k=1,2, \cdots, K,
$$

where $\angle$ (.) returns the angle of any complex number. Finally, recall that for the $\left\{l^{t h}\right\}_{l=1}^{N_{a}-2 k-1}$ column, we have $P_{l}+1=N_{a}-2 K-l$ estimates for the same DOA $\theta_{k}$, which means that by considering all the eligible columns, we have

$$
\sum_{l=1}^{N_{a}-2 K-1}\left(N_{a}-2 K-l\right)=\frac{\left(N_{a}-2 K-1\right)\left(N_{a}-2 K\right)}{2}
$$

estimates for each DOA. Therefore, one can average over all these estimates (obtained column wise) to obtain more refined estimates for the unknown DOAs as follows:

$$
\begin{aligned}
& \hat{\theta}_{k}^{\text {column }}= \\
& \frac{2}{\left(N_{a}-2 K-1\right)\left(N_{a}-2 K\right)} \sum_{l=1}^{N_{a}-2 K-1} \sum_{p=0}^{N_{a}-l-2 K-1} \hat{\theta}_{k}^{(l, p)} .
\end{aligned}
$$

So far, we have used all the first $N_{a}-2 K-1$ columns. Yet, the remaining columns $\left(l \geq N_{a}-2 K\right)$ can also be exploited to further refine the DOA estimates. This may seem a priori impossible since these columns-or equivalently the corresponding vectors $\left\{\boldsymbol{r}_{\boldsymbol{\theta}}^{(l)}\right\}_{l=N_{a}-2 K}^{N_{a}}$-do not indeed contain the necessary $2 K+1$ cross-covariances as previously required. Yet, the elements of these columns belong to the last $N_{a}-2 K-1$ rows that contain necessarily more than $2 K+1$ adjacent covariances. Indeed, recalling that the covariance matrix $\bar{\Sigma}(\boldsymbol{y})$ is Toeplitz structured, it becomes clear that the last $N_{a}-2 K-1$ rows can also be exploited in the same way providing thereby a new set of estimates for the DOAs. To that end, for the $\left\{l^{t h}\right\}_{l=2 K+2}^{N_{a}}$ row, we construct the corresponding vectors $^{6}, \boldsymbol{r}_{\boldsymbol{\theta}}^{((l)}=\left[r_{\boldsymbol{\theta}}^{\prime(l)}(1), r_{\boldsymbol{\theta}}^{(l)}(2), \cdots, r_{\boldsymbol{\theta}}^{\prime(l)}(l-1)\right]^{T}$ whose $m^{\text {th }}$ element is defined as

$$
r_{\boldsymbol{\theta}}^{\prime(l)}[m]=\Sigma_{\boldsymbol{y}}(l, l-m), m=1,2, \cdots, l-1 .
$$

Therefore, for each $l=N_{a}, N_{a}-1, \cdots, 2 K+2$, the sequence $\left\{r_{\theta}^{\prime(l)}[m]\right\}_{m=1}^{l-1}$ inherits the important structure of linear combinations of weighted exponentials. Then, applying the same procedure using the vectors $\boldsymbol{r}_{\boldsymbol{\theta}}^{(l)}$ instead of $\boldsymbol{r}_{\boldsymbol{\theta}}^{(l)}$, we obtain an additional row-wise refined estimate for each DOA which we denote $\hat{\theta}_{k}^{\text {row }}$. Lastly, the final estimates of the DOAs are obtained as

$$
\hat{\theta}_{k}=\frac{\hat{\theta}_{k}^{\text {column }}+\hat{\theta}_{k}^{\text {row }}}{2}, k=1,2, \cdots, K .
$$

Based on the analysis so far introduced, it may seem that the new estimator works only if an extremely large number of snapshots are available at the receiver side. In fact, all the derivations are based on the theoretical expression of the elementary cross-covariances, $\Sigma_{y}(i, l)$, given in (5) although in practice these elementary cross-covariances are estimated by sample averaging as follows:

$$
\widehat{\Sigma}_{\boldsymbol{y}}(i, l)=\frac{1}{N} \sum_{n=1}^{N} y_{i}(n) y_{l}^{H}(n)
$$

and this sample average does not coincide with the statistical average given in (5) unless the observation window size, $N$, is very large. Yet, we will see in the simulations section that the new AF-based estimator performs very well with very short-data records and even from a single snapshot. 


\subsection{Robustness to the presence of short data records}

The resilience to the presence of short data records can be proven theoretically. In this analysis, we considers the case of short data records but it is also valid for the single-snapshot scenario by simply taking $N=1$. For convenience, we adopt the notation $\widehat{\Sigma}_{y}^{l}(i)$ for the estimated elementary cross-covariances of (25) instead of $\widehat{\Sigma}_{y}(i, l)$, which are given by

$$
\widehat{\Sigma}_{\boldsymbol{y}}^{l}(i)=\frac{1}{N} \sum_{n=1}^{N} y_{i}(n) y_{l}(n)^{*},
$$

and we show that the unknown DOAs can still be estimated from the roots of the corresponding annihilating filter. In fact, we have for $n=1,2, \cdots, N$ :

$$
\begin{aligned}
& y_{i}(n) y_{l}(n)^{*}= \\
& \quad\left(\sum_{k=1}^{K} h_{k} a_{k}(n) e^{j(i-1) \pi \sin \left(\theta_{k}\right)}\right) y_{l}(n)^{*}+w_{i}(n) y_{l}(n)^{*} .
\end{aligned}
$$

Then, for medium and high SNR values, the noise components are small compared to the useful signal component, i.e. $\left|w_{i}(n)\right| \ll\left|\sum_{k=1}^{K} h_{k} a_{k}(n) e^{j(i-1) \pi \sin \left(\theta_{k}\right)}\right|$ with very high probability meaning that

$$
\left|\sum_{k=1}^{K} h_{k} a_{k}(n) e^{j(i-1) \pi \sin \left(\theta_{k}\right)} y_{l}(n)^{*}\right| \ll\left|w_{i}(n) y_{l}(n)^{*}\right| .
$$

Therefore, the second term in (27) (stemming from the noise component) can be reasonably neglected, and we obtain an approximate expression for the estimated covariances between antenna element $i$ and antenna element $l$, as follows:

$$
\begin{aligned}
\widehat{\Sigma}_{y}^{l}(i) & =\frac{1}{N} \sum_{n=1}^{N} y_{i}(n) y_{l}(n)^{*}, \\
& \approx \frac{1}{N} \sum_{n=1}^{N} y_{l}(n)^{*}\left(\sum_{k=1}^{K} h_{k} a_{k}(n) e^{j(i-1) \pi \sin \left(\theta_{k}\right)}\right) \\
& =\frac{1}{N} \sum_{n=1}^{N} \sum_{k=1}^{K} \underbrace{y_{l}(n)^{*} h_{k} a_{k}(n)}_{g_{k, l}(n)} e^{j(i-1) \pi \sin \left(\theta_{k}\right)} \\
& =\frac{1}{N} \sum_{n=1}^{N} \sum_{k=1}^{K} g_{k, l}(n) e^{j(i-1) \pi \sin \left(\theta_{k}\right)} \\
& =\sum_{k=1}^{K}(\underbrace{\frac{1}{N} \sum_{n=1}^{N} g_{k, l}(n)}_{G_{k, l}} u_{k}^{i-1} . \\
& =\sum_{k=1}^{K} G_{k, l} u_{k}^{i-1} .
\end{aligned}
$$

We observe from (29) that the second-order moments estimated with short data records (or even a single snapshot) exhibit the interesting property of a "weighted sum of sinusoids" and therefore the DOAs can still be accurately estimated from the roots of their annihilating filter.

\subsection{Exploiting the Toeplitz structure of the covariance matrix}

When the propagation conditions are very harsh, the SNR experienced at the receiver side can be very low. In this scenario, the received signals are too much corrupted by the noise components and therefore the estimated cross-covariances are very noisy, especially from short data records. Hence, exploiting the fact that the covariance matrix is Toeplitz structured, one can average along the secondary diagonals in order to obtain a set of more accurate estimates for the cross-covariances. The DOA estimation is then performed in the same way using the new single sequence of more accurate $N_{a}$ covariances. In fact, we see from (5) that for $m=1,2, \cdots, N_{a}-1$

$\Sigma_{y}(l+m, l)=\Sigma_{y}(1+m, 1), l=1,2, \cdots, N_{a}-m$.

Therefore, for each fixed lag $m,\left\{\widehat{\Sigma}_{y}(l+m, l)\right\}_{l=1}^{N_{a}-m}$ can be averaged as follows to obtain the following more refined statistics:

$$
\begin{aligned}
& \widehat{\bar{r}}_{\theta}(m)= \\
& \frac{1}{N_{a}-m} \sum_{l=1}^{N_{a}-m} \widehat{\Sigma}_{y}(l+m, l), m=1,2, \cdots, N_{a}-1,
\end{aligned}
$$

from which we construct a single vector, $\widehat{\overline{\boldsymbol{r}}}_{\theta}=$ $\left[\widehat{\bar{r}}_{\theta}(1), \widehat{\bar{r}}_{\theta}(2), \cdots, \widehat{\bar{r}}_{\theta}\left(N_{a}-1\right)\right]^{T}$. Then, the same procedure that was previously applied for all the eligible columns is now applied to the single vector $\widehat{\vec{r}}_{\theta}$ since it also inherits the interesting property of weighted sum of exponentials. For ease of notation, we simply refer to this procedure as version II of the new AF-based estimator and we refer to the procedure described previously (column-wise and row-wise) as version $I$.

This operation of averaging over the secondary diagonals is not only useful to combat the effect of the noise at low SNRs but also expected to improve the DOA estimation even for moderate SNR values whenever the number of sources to be localized is large. In fact, when $K$ is high, the number $N_{a}-2 K-1$ of eligible columns in version $I$ can be limited. For instance, for $N_{a}=8$ and $K=3$, only the first column is eligible since $N_{a}-2 K-1=1$. Consequently, a large part of the covariance matrix is simply ignored although it carries a lot of information about the unknown DOAs. Yet, by averaging over the secondary diagonals, all the entries of the covariance matrix are incorporated in the estimation process and the 
whole information is being exploited. Therefore, as long as the SNR decreases or the number of sources increases (for a fixed number of receiving antenna elements), it is expected that the second version of the new estimator outperforms its first version. However, for sufficiently high SNR values, the estimated elementary cross-covariances (without averaging) are already quite accurate and can hence be reliably used to obtain more accurate ${ }^{7}$ DOA estimates with version $I$. The latter is even more recommended if the number of sources is also small since the number of eligible columns (and consequently the number of exploited cross-covariances) would be sufficiently high.

\subsection{Complexity analysis}

In this subsection, we assess the complexity of the new estimator vs. the other single-snapshot techniques ${ }^{8}$. To that end, we evaluate the number of operations (additions and multiplications) required by each estimator. In particular, the new estimator involves two major steps which are (i) the estimation of the covariance matrix that requires $N N_{a}\left(N_{a}-1\right)$ operations and (ii) the SVD decomposition and polynomial rooting procedures which require $2\left(N_{a}-2 K-1\right)\left(N_{a}-2 K\right) O\left(K^{3}\right)$ operations. Of course, it involves also at the very end a simple step in which the individual estimates are averaged requiring $\left(N_{a}-2 K-1\right)\left(N_{a}-2 K\right)$ extra operations. On the other hand, the overall complexities of the DML and Bayesian estimators are $R^{K}\left(2 N_{a}^{3}+(N+2 K-1) N_{a}^{2}+\left(4 N_{a}-1\right) K^{2}-\left(N+N_{a}\right)\right.$ $\left.K+O\left(K^{3}\right)\right)$ and $R^{K}\left((4 K+3) N_{a}^{2}+\left(12 N_{a}-3\right) K^{2}-\right.$ $\left.(3 K-2) N_{a}+3 O\left(K^{3}\right)\right)$, respectively, where $R$ is the number of samples on the parameters grid corresponding to a discretization step, $s$, of $s=180 / R$. Notice here that the complexity of these two traditional estimators grows exponentially with the number of unknown DOAs, $K$, as reflected by the multiplicative term $R^{K}$. It comes clear now that increasing $R$ (i.e. considering a denser grid search for more refined estimates) increases prohibitively their computational cost. Typically, for $N_{a}=16, N_{s}=2$, $N=1$, and $R=100$, the total number of operations performed by our AF-based method, to estimate all the DOAs, is about 2484 operations. However, to evaluate their objective functions just at a single search point $\left(\theta_{i}, \theta_{j}\right)$ in the grid, the Bayesian and DML estimators require about 3532 and 9442 operations, respectively, i.e. already far more than the overall complexity of our estimator. To find the estimates of the DOAs as the maximum of their objective functions over all the grid points, these two classical estimators require in total as much as $100^{2} \times 3532=35.32 \times 10^{6}$ and $100^{2} \times 9442=94.42 \times 10^{6}$ operations against just 2484 operations with the proposed estimator. Of course, the performance of these gridsearch estimators improves constantly as $R$ increases, but their computational load becomes prohibitively very high. This is illustrated in Table 1 where we present the computational load of the three estimators in different setups by evaluating their complexities at various values for the couple $(K, R)$ with a fixed array-size of $N_{a}=16$. It is clearly seen from this table that our estimator is far less computationally expensive than both existing single-snapshot techniques. Moreover, it will be shown later through computer simulations that it outperforms both of them in accuracy over a large SNR range.

\section{Per-source channel power estimation}

The per-source estimation of the channel power (or signal power) is very useful in wireless communication systems. In fact, in a multi-user mobile communication systemwhere each active user can be seen as an active sourcethe knowledge of this parameter is used to manage the interference. For instance, a well-known scheme for multiuser detection is the successive interference cancellation (SIC). The scheme is based on demodulating the strongest interferer and removing its effect from the received signal [15] and then continuing this procedure for the next strongest user. Because of its simplicity and also its capability in combating strong interferers [15], SIC is the subject of great attention for practical systems design; see [16-18] and references therein. Clearly, SIC requires the power of the strongest interferer at each stage of interferer cancellation. There are of course many other useful applications beyond this example that justify the need of properly estimating both the source and noise powers and the SNR. Motivated by this fact, we exploit the DOA estimates provided by our AF-based technique in order to estimate the individual channel powers almost instantaneously over a very short data records $(N<10$ for instance). To that end, we take the first $K$ averaged covariances in (31), $\bar{r}_{\theta}[m]=\sum_{k=1}^{K}|h(k)|^{2} u_{k}^{m}, m=1,2, \cdots, K$, from which we write the following matrix system:

$$
\underbrace{\left(\begin{array}{c}
\bar{r}_{\boldsymbol{\theta}}[1] \\
\bar{r}_{\boldsymbol{\theta}}[2] \\
\vdots \\
\bar{r}_{\boldsymbol{\theta}}[K]
\end{array}\right)}_{\overline{\boldsymbol{r}}_{\boldsymbol{\theta}}}=\underbrace{\left(\begin{array}{ccccc}
u_{1} & u_{1}^{2} & u_{1}^{3} & \cdots & u_{1}^{K} \\
u_{2} & u_{2}^{2} & u_{2}^{3} & \cdots & u_{2}^{K} \\
\vdots & \vdots & \ddots & \ddots & \vdots \\
u_{K} & u_{K}^{2} & \cdots & \cdots & u_{K}^{K}
\end{array}\right)}_{\boldsymbol{U}(\boldsymbol{\theta})} \underbrace{\left(\begin{array}{c}
\left|h_{1}\right|^{2} \\
\left|h_{2}\right|^{2} \\
\vdots \\
\left|h_{K}\right|^{2}
\end{array}\right)}_{\boldsymbol{h}} .
$$

Table 1 Complexity of the three single-shot techniques with $N_{a}=16$ receiving antenna branches

\begin{tabular}{|c|c|c|c|c|}
\hline & \multicolumn{2}{|c|}{$K=2$} & \multicolumn{2}{|c|}{$K=4$} \\
\hline & $R=100$ & $R=500$ & $R=100$ & $R=500$ \\
\hline \multicolumn{3}{|c|}{ Bayesian method $35.32 \times 10^{6} 8.83 \times 10^{8}$} & $7.9200 \times 10^{11}$ & $4.95 \times 10^{14}$ \\
\hline DML & $94.42 \times$ & $62.3605 \times 1$ & $91.1244 \times 10^{12}$ & $7.0275 \times 10^{14}$ \\
\hline AF-based & 2484 & 2484 & 7464 & 7464 \\
\hline
\end{tabular}


Now injecting the estimated DOAs, $\widehat{\theta}_{k}$, in $u_{k}$ instead of the true DOAs, $\theta_{k}$, we construct an estimated matrix, $\widehat{\boldsymbol{U}}(\boldsymbol{\theta})$, using $\widehat{u}_{k}=e^{j \pi \sin \left(\widehat{\theta}_{k}\right)}$, to substitute $\boldsymbol{U}(\boldsymbol{\theta})$ in the system (32) in which the only remaining unknowns are $\left\{\left|h_{k}\right|^{2}\right\}_{k=1}^{K}$. Thus, by inverting $\widehat{\boldsymbol{U}}(\boldsymbol{\theta})$ and using $\widehat{\overline{\boldsymbol{r}}}_{\boldsymbol{\theta}}$ instead of $\overline{\boldsymbol{r}}_{\boldsymbol{\theta}}$, one can easily obtain a joint estimate, $\widehat{\boldsymbol{h}}=$ $\left[\left|\widehat{h}_{1}\right|^{2},\left|\widehat{h}_{2}\right|^{2}, \cdots,\left|\widehat{h}_{K}\right|^{2}\right]^{T}$, for the channel powers, $\boldsymbol{h}=$ $\left[\left|h_{1}\right|^{2},\left|h_{2}\right|^{2}, \cdots,\left|h_{K}\right|^{2}\right]^{T}$, as follows:

$$
\widehat{\boldsymbol{h}}=\widehat{\boldsymbol{U}}(\boldsymbol{\theta})^{-1} \overline{\boldsymbol{r}}_{\boldsymbol{\theta}} .
$$

Note that $\widehat{\boldsymbol{U}}(\boldsymbol{\theta})$ is a Vandermonde matrix and therefore its inverse always exists as far as the DOAs are different (resolvable DOAs). Now after obtaining the estimates of $\left\{\left|h_{k}\right|^{2}\right\}_{k=1}^{K}$, we extract an estimate of the noise power from the main diagonal elements of the covariance matrix. In fact, we see from (5) that for the $\left\{l^{t h}\right\}_{l=1}^{N_{a}}$ diagonal element, we have

$$
2 \sigma^{2}=\Sigma_{y}(l, l)-\sum_{k=1}^{K}\left|h_{k}\right|^{2} .
$$

Therefore, using the estimates $\left|\widehat{h}_{k}\right|^{2}$ of $\left|h_{k}\right|^{2}$ obtained in (33), we obtain an estimate $\widehat{\sigma}_{l}^{2}$ of $\sigma^{2}$ for each $l=$ $1,2, \cdots, N_{a}$ as follows:

$$
\widehat{\sigma}_{l}^{2}=\frac{1}{2}\left(\widehat{\Sigma}_{y}(l, l)-\sum_{k=1}^{K}\left|\widehat{h}_{k}\right|^{2}\right), l=1,2, \cdots, N_{a},
$$

which can be averaged over all the possible values of $l$ to obtain a more accurate estimate of the noise variance:

$$
\widehat{\sigma^{2}}=\frac{1}{N_{a}} \sum_{l=1}^{N_{a}} \widehat{\sigma}_{l}^{2}=\frac{1}{2 N_{a}} \sum_{l=1}^{N_{a}} \widehat{\Sigma}_{\boldsymbol{y}}(l, l)-\frac{1}{2} \sum_{k=1}^{K}\left|\widehat{h}_{k}\right|^{2} \text {. }
$$

Finally, using (33) and (36), an estimate of the SNR of each source is simply given by

$$
\widehat{\rho}_{k}=\frac{\left|\widehat{h}_{k}\right|^{2}}{2 \widehat{\sigma}^{2}} \text {. }
$$

\section{Simulation results}

In this section, we assess the performance of the new DOA estimator using the mean square error (MSE) as a performance measure. The MSE is computed for each estimator $\hat{\theta}_{k}$ of the $k^{\text {th }}$ DOA, $\theta_{k}$, as follows:

$$
\operatorname{MSE}\left(\hat{\theta}_{k}\right)=\frac{1}{M_{c}} \sum_{q=1}^{M_{c}}\left(\hat{\theta}_{k}^{(q)}-\theta_{k}\right)^{2},
$$

where $M_{c}$ is the number of Monte-Carlo simulations which is set to $M_{c}=1000$ in all simulations and $\hat{\theta}_{k}^{(q)}$ is the estimate of $\theta_{k}$ from the $q^{\text {th }}$ Monte-Carlo run. We also consider the well-known root-MUSIC (RM) estimator and the Cramér-Rao lower bound (CRLB) [10] as a benchmark against which we compare the performance of our newly developed method in the case of a large number of snapshots. In the case of short data records, we also add the Multi-Task Bayesian Compressed Sensing (MT-BCS) technique [19] as a benchmark. We propose also another performance metric where we show the resolution probabilities for the AF-based and root MUSIC techniques. In the more challenging case where a single snapshot is available at the receiver side, we compare our method to the Bayesian estimator [12] and the Single Task Bayesian Compressed Sensing (ST-BCS) technique [19] that are both specifically designed to cope with this extreme scenario. We also compare it to the deterministic ML estimator that is recognized to be the most accurate in this case [14]. For the sake of conciseness, we consider without loss of generality the case of equipowered sources and provide simulation results only for the first source (DOA and channel power). Yet, we emphasize the fact that the same performance behaviour can be observed from the other sources. For the channel power estimator, we adopt the normalized root mean square error (NRMSE) as a performance measure defined as

$$
\operatorname{NRMSE}\left(\left|\widehat{h}_{k}\right|^{2}\right)=\frac{\sqrt{\operatorname{MSE}\left(\left|\widehat{h}_{k}\right|^{2}\right)}}{\left|h_{k}\right|^{2}} .
$$

The NRMSE for the SNR estimator is defined likewise. DOA estimation will be basically organized in three subsections: (i) the case of multiple snapshots (including short-data records), (ii) the case of a single-shot measurement, and (iii) the case of time varying DOAs. Channel powers and SNR estimation will then follow.

\subsection{Multiple and short-data records: comparison against root-MUSIC}

In Fig. 1, we plot for the three estimators (version $I$ and verion II of the AF-based and root-MUSIC) the MSE of the DOA estimates for the first source obtained from $N=1000$ received samples, with $N_{a}=8$ and $N_{a}=16$ receiving antenna elements, versus the SNR of the same source.

We see that the two versions of the new estimator provide sufficiently accurate DOA estimates over the entire SNR range. In such comfortable situation where a very large number of measurements can be used in the estimation process, the classical root-MUSIC technique outperforms the two AF-based versions. It is also seen that as $N_{a}$ increases, version I of the AF-based estimator exhibits a performance gain against its version $I I$ at low SNR values. Actually, this is only true when the window size is large enough (e.g. $N=1000$ as considered in this figure) so that the elementary cross-covariances are quite accurate and therefore the elementary estimates $\hat{\theta}^{(l, k)}$ are also sufficiently accurate. Indeed, since the number of these elementary estimates $\left(N_{a}-2 K-1\right.$ eligible columns and rows) also increases with $N_{a}$, this leads to a more accurate final averaged estimate than the single estimate obtained 

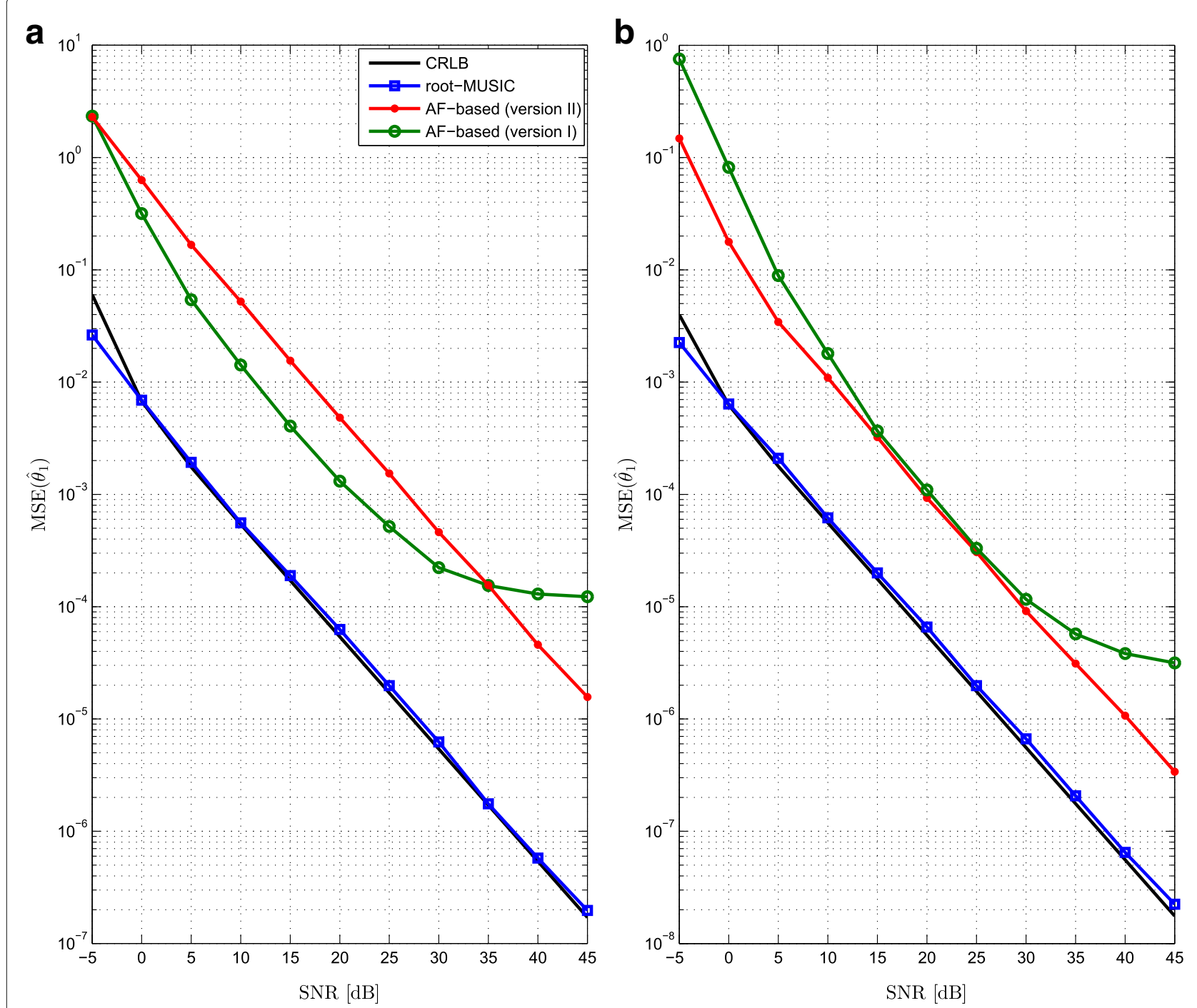

Fig. 1 MSE for the first DOA versus the SNR with $K=2$ sources, $N=1000$ snapshots, $\theta_{1}=18^{\circ}$, and $\theta_{2}=36^{\circ}$ : (a) $N_{a}=8$ antenna elements and (b) $N_{a}=16$ antenna elements

by applying version II. The same observation holds for sufficiently high SNR values even if $N_{a}$ is small $\left(N_{a}=8\right)$.

In Fig. 2, we simulate a more adverse situation in which the DOAs are estimated from a very limited number of snapshots $(N=3$ for example). The major advantage of our new estimator is now revealed. In fact, both versions of the new technique outperform by far, in terms of estimation accuracy, the RM estimator with an advantage for version II over version $I$ (the advantage of exploiting the Toeplitz structure is now clearer). Yet, the former's performance saturates at very high SNR values whereas the latter's improves linearly with the SNR. The MT-BCS technique shows in Fig. 2 good performance in the case of short data records (i.e. $N=3$ ). Unfortunately, its computational complexity is dictated by the grid discretization step, and a trade-off between complexity and performance must be made.[19].

In Fig. 3, we provide the probability of separation versus the angular separation and the SNR. The probability of separation, as defined in [20], states that two signals are said to be resolved if their respective DOA estimates, $\hat{\theta}_{1}$ and $\hat{\theta}_{2}$, are such that $\left|\hat{\theta}_{1}-\theta_{1}\right|<\left|\theta_{1}-\theta_{2}\right| / 2$ and $\left|\hat{\theta}_{2}-\theta_{2}\right|<\left|\theta_{1}-\theta_{2}\right| / 2$. The root-MUSIC technique outperforms the two versions of the annihilating filter technique at extremely low angle separations. However, the AF technique succeeds in resolving over $95 \%$ of the possible cases starting from a separation of $\Delta \theta=10^{\circ}$. Clearly, AF-version II has a better resolution performance with closely spaced angles. Figure $3 b$ depicts the probabilities of resolution as function of the SNR values. Both AF 


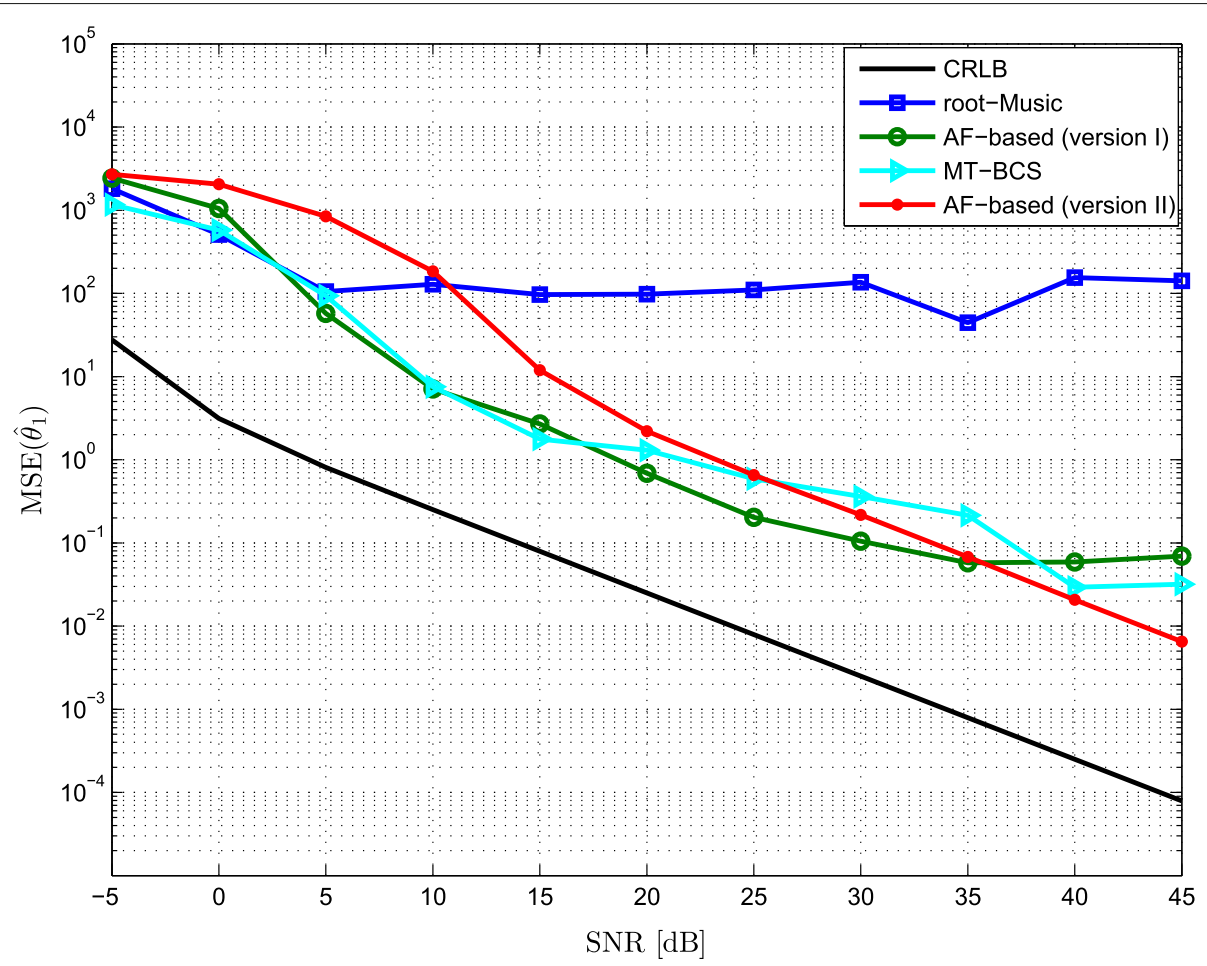

Fig. 2 MSE for the first DOA versus the SNR with $K=2$ sources, $N=3$ snapshots, $\theta_{1}=18^{\circ}, \theta_{2}=36^{\circ}$, and $N_{a}=8$ antenna elements

techniques succeed in resolving the sources in $90 \%$ of the cases starting from the SNR value of $3 \mathrm{~dB}$.

\subsection{Single-shot case: comparison against the DML and Bayesian methods}

Now, we consider a situation which is even more challenging where we assume that the receiver needs to estimate the DOAs from a single snapshot. In this situation, we compare the performance of our method against that of three estimators that are tailored specifically to the singleshot case: the Bayesian method of [12], the single-shot DML estimator [14], and ST-BCS. We recall that, according to the recent survey of [14], the DML technique stands as the most accurate among various single-shot estimators. We plot in Fig. 4 the MSE for the four estimators for $N=1$ (i.e. only one sample is available at the receiver side) and $N_{a}=16$ receiving antenna branches.

The three existing estimators were simulated using a discretization step $s=180 / 100$ (in the remainder of this paper, we will characterize the grid step, $s$, by the integer number $R$ where $s=180 / R$ ). We observe from this figure that both versions of the newly developed AF-based estimator are still able to estimate the DOAs over a wide SNR range.

We see also from Fig. 4 that for sufficiently high SNR values the MSE of version II saturates, contrarily to version $I$. This is because in this SNR region the signals are almost noise-free and therefore the elementary crosscovariances' estimates are already noiseless. They can be thus exploited as they are (as done in version I) to provide a large number of sufficiently accurate estimates $\hat{\theta}_{k}^{(l, p)}$ without prior averaging (as done in version $\left.I I\right)$. In fact, averaging along the secondary diagonals would simply provide a number of statistics that are as accurate as the elementary cross-covariances themselves, and hence, the performance in terms of DOA estimation does not improve (saturation).

On the other hand, the existing single-shot techniques (Bayesian, DML estimators and ST-BCS) exhibit a slight advantage at low SNR levels, but their computational load is extremely much higher. In fact, in light of the complexity analysis presented in Table 1 at the end of Section 3.3, the complexities of the DML and Bayesian algorithms are, respectively, in the order of $N_{\text {oper }}^{\text {Bayesian }}=35.32 \times 10^{6}$ and $N_{\text {oper }}^{\mathrm{DML}}=94.42 \times 10^{6}$ operations against only $N_{\text {oper }}^{\mathrm{AF}}=2484$ operations for the proposed estimator. This amounts to complexity ratios in the order of $\frac{N_{\text {oper }}^{\text {Bayesian }}}{N_{\text {oper }}^{\mathrm{AF}}} \approx \frac{N_{\text {oper }}^{\mathrm{DML}}}{N_{\text {oper }}^{\mathrm{AF}}} \approx 10^{4}$. Yet, even at these extremely high computational loads, the traditional single-snapshot algorithms are not able to outperform the new estimator for medium to high levels. Of course, as stated previously, for extremely large values of $R$ (very dense grid search), these two estimators would ultimately outperform our new method over the entire SNR 

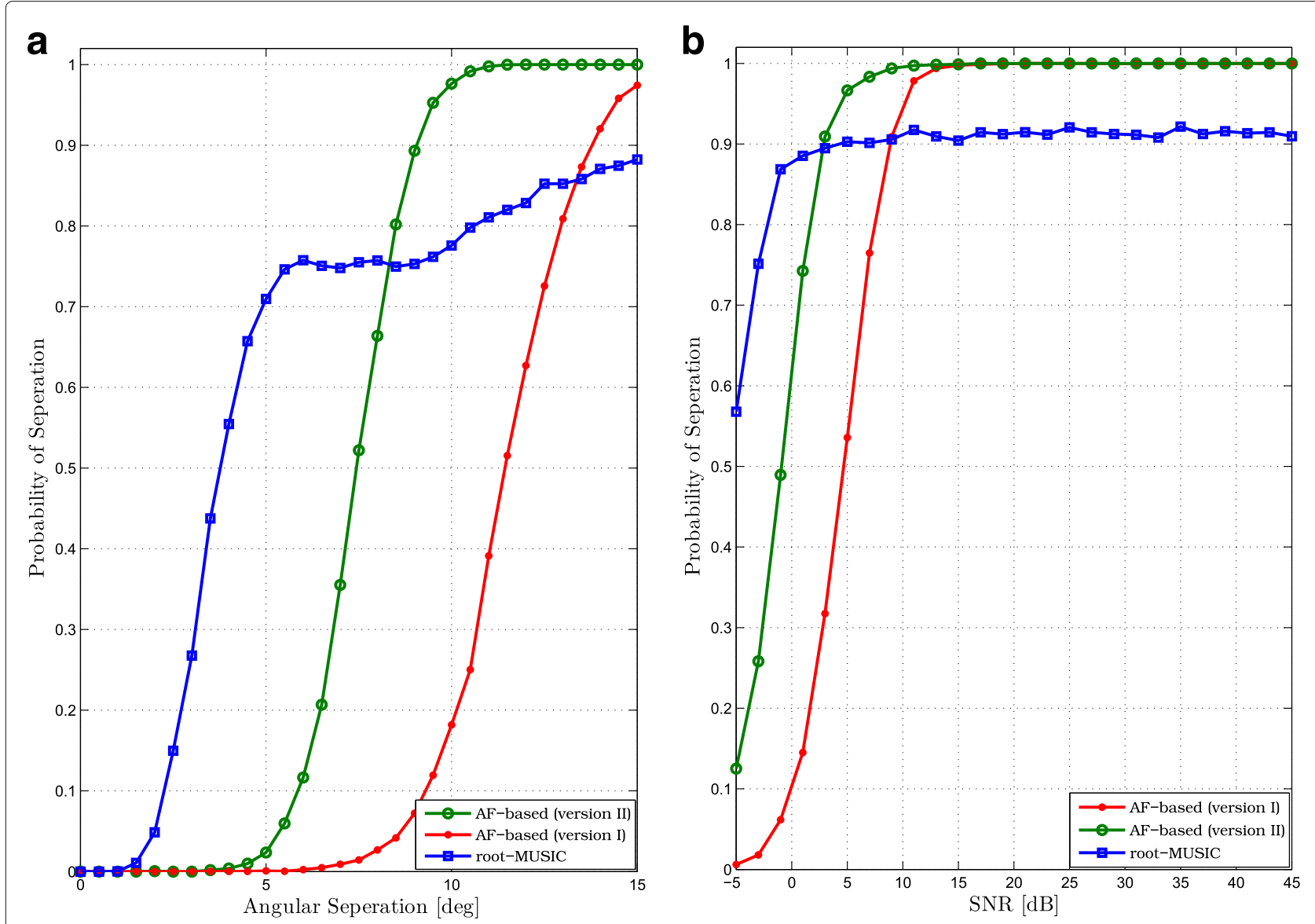

Fig. 3 Probability of resolution (a) versus angular separation $(N=3$ and $S N R=15 \mathrm{~dB})$ and $(\mathbf{b})$ versus $S N R\left(\Delta \theta=15^{\circ}\right.$ and $\left.N=3\right)$

range, but unfortunately their complexities become even more prohibitive 9 . For example, under the same simulation setup of Fig. 4 (in particular $N_{a}=16$ and $K=2$ ), these two estimators will outperform the AF-based technique, over the entire SNR range, by setting $R=500$ (i.e. estimating the DOAs at a grid resolution of $0.36^{\circ}$ ). However, the complexity ratios become in the order of $\frac{N_{\text {oper }}^{\text {Bayesian }}}{N_{\text {oper }}^{\mathrm{AF}}} \approx \frac{N_{\text {oper }}^{\mathrm{DML}}}{N_{\text {oper }}^{\mathrm{AF}}} \approx 10^{6}$.

The new method is therefore very useful (in terms of accuracy/complexity trade-offs) in applications where a single snapshot is to be used. This is encountered in many situations where a very high estimation update speed is required. These applications can be indeed enhanced by providing a DOA estimate once a single sample is acquired instead of waiting for a larger number of measurements. Furthermore, in many other practical situations, the DOAs may change appreciably from one snapshot to another due to the fast motion of the sources. For all these systems, our new AF-based estimator offers the best accuracy/complexity trade-offs.

\subsection{Time-varying DOAs}

To illustrate the time-varying DOA tracking capability of the new AF-based estimator, we plot in Fig. 5 the estimated DOAs using its version $I I$ and the DML algorithm for two moving sources and an SNR level of $15 \mathrm{~dB}$. The DOAs were generated assuming that both sources increase linearly from $-60^{\circ}$ and $-30^{\circ}$, respectively, with a radial speed $\dot{\theta}_{1}=\dot{\theta}_{2}$ as high as $1.175^{\circ}$ per sample, over 80 data snapshots. Both estimators were applied using $N=1$ (i.e. single snapshot). It is seen that both AF and DML estimates follow accurately the trajectories of the two time-varying DOAs. Yet, as depicted in Fig. 6, the AF-based estimator exhibits lower tracking error at significantly much less computational cost. Furthermore, since the new estimator performs well with a single data snapshot, its tracking performance will prove the same no matter the angular speed ranges of the DOA time variations reach.

Now coming back to the root-MUSIC estimator, recall that it was assessed from Fig. 1 to outperform our new AFbased estimator for large window sizes. This was for constant DOAs. Actually, for time-varying DOAs, depending 


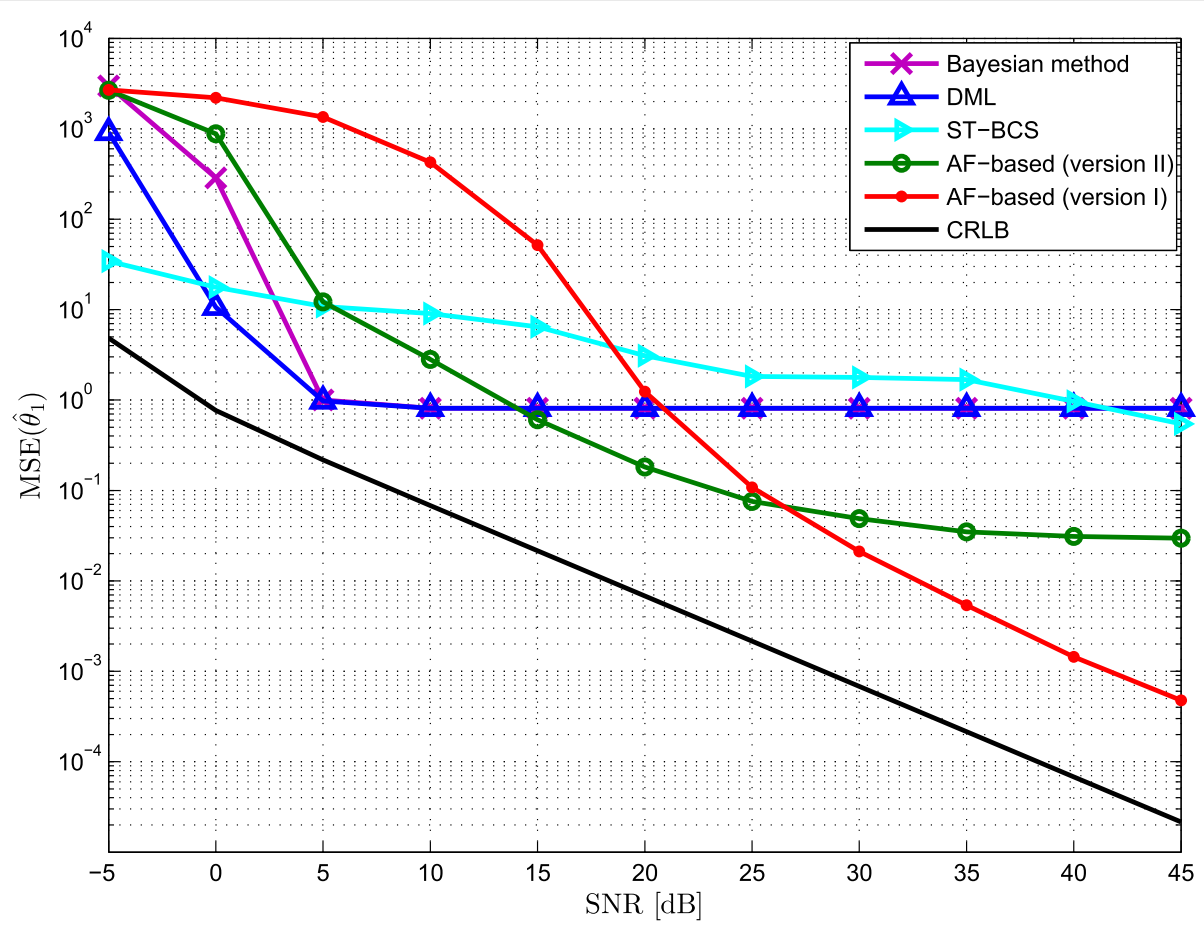

Fig. 4 MSE for the first DOA versus the SNR with $K=2$ sources, $N=1, \theta_{1}=20.7^{\circ}, \theta_{2}=40.5^{\circ}, N_{a}=16$ antenna elements, and $16-Q A M$

on the angular speed range, the AF-based estimator might outperform root-MUSIC even when data records are not short. In fact, when the DOAs are not constant over a time period, applying the root-MUSIC algorithm locallyover an observation window of short size-would simply return an estimate of the average DOAs over this considered window. Clearly, in this case, the performance of the root-MUSIC algorithm is affected by the size of the local window and the DOAs speed. Indeed, as speed increases, the DOAs tend to vary appreciably within the processing window duration and, hence, the performance of the root-MUSIC estimator degrades as the approximation of locally constant DOAs becomes increasingly inaccurate. Our new estimator, however, does not suffer from this drawback since it succeeds in accurately estimating the DOAs from very short data records and since it is also very robust to fast DOA time variations (as seen from Figs. 2 and 5). This behaviour is illustrated in Fig. 7 where we show the operational regions, in terms of window sizes $(N)$ and DOA speeds $(\dot{\theta})$, for the two estimators. A region is attributed to a given estimator when this estimator shows lower MSE for all the couples $(N, \dot{\theta})$ in this region. We see that when the DOAs vary so rapidly, our new estimator outperforms the RM technique even in the case of multiple snapshots (upper right corner of Fig. 7) contrarily to what was observed in Fig. 1 where the DOAs where assumed constant (which corresponds to $\dot{\theta}=0^{\circ}$ /sample and $N=1000)$.

\subsection{Performance of the channel powers and SNR estimators}

Here, we assess the performance of the channel power estimator derived in section IV. For two different values of the observation window sizes, namely $N=10$ and $N=1000$, we plot in Figs. 8 and 9, respectively, the NRMSE for the channel power estimator using both versions $I$ and $I I$ as a function of the true SNR. First, notice from Fig. 8 that the channel power is estimated quite accurately using only few received samples, $N=10$ snapshots, especially in the moderate/high experienced SNR values. Naturally, the estimation accuracy is enhanced in Fig. 9 for a larger window size, i.e. $N=1000$ where both versions provide very accurate estimates for the channel power, a key parameter that is often used for the design of wireless communication schemes. We also observe from these two figures, for these large antenna array-sizes $\left(N_{a}=16\right.$ and $N_{a}=32$ ), the performance improvements of version $I$ against version $I I$ at low SNR values, a fact that is mainly due to the improvements in DOA estimation in this region as explained previously (see comments on Fig. 1).

Lastly, Fig. 10 depicts the NRMSE for both SNR estimators as function of the true SNR values for different values of $N_{a}$. It is seen from this figure that performance improves by increasing $N_{a}$, which is hardly surprising. Yet, the SNR estimates are not as quite accurate as those of the channel strength. This stems mainly from the estimation error on the noise variance. At a first sight, one would 

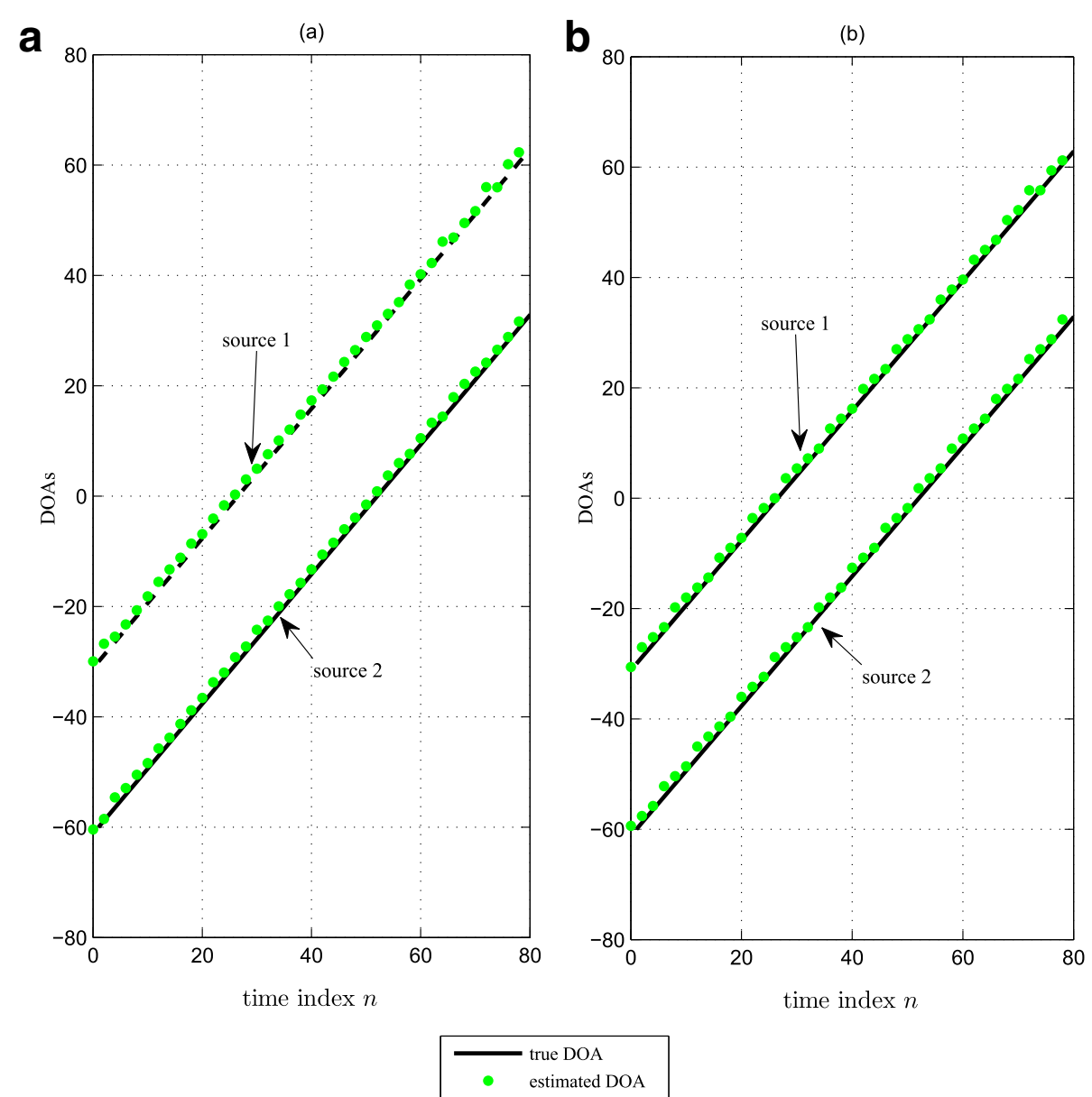

Fig. 5 True DOAs and their estimates with QPSK, $K=2$ sources, $N=1, \dot{\theta}_{1}=\dot{\theta}_{2}=1.175^{\circ} /$ sample, $R=100, N_{a}=16$ and SNR=15 dB: (a) AF-based (version II) and (b) DML

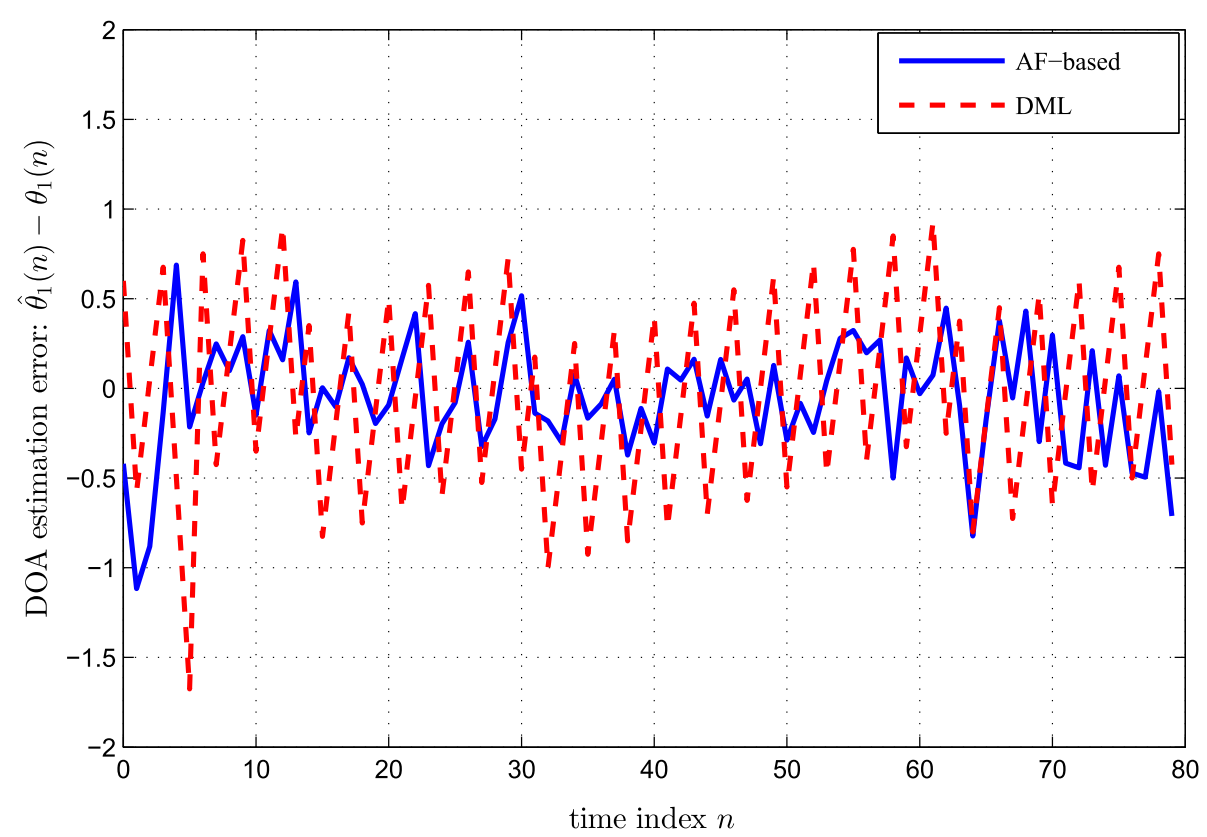

Fig. 6 Tracking error of both estimators with QPSK, $K=2$ sources, $N=1, \dot{\theta}_{1}=\dot{\theta}_{2}=1.175^{\circ} /$ sample, $R=100, N_{a}=16$, and SNR=15 dB 


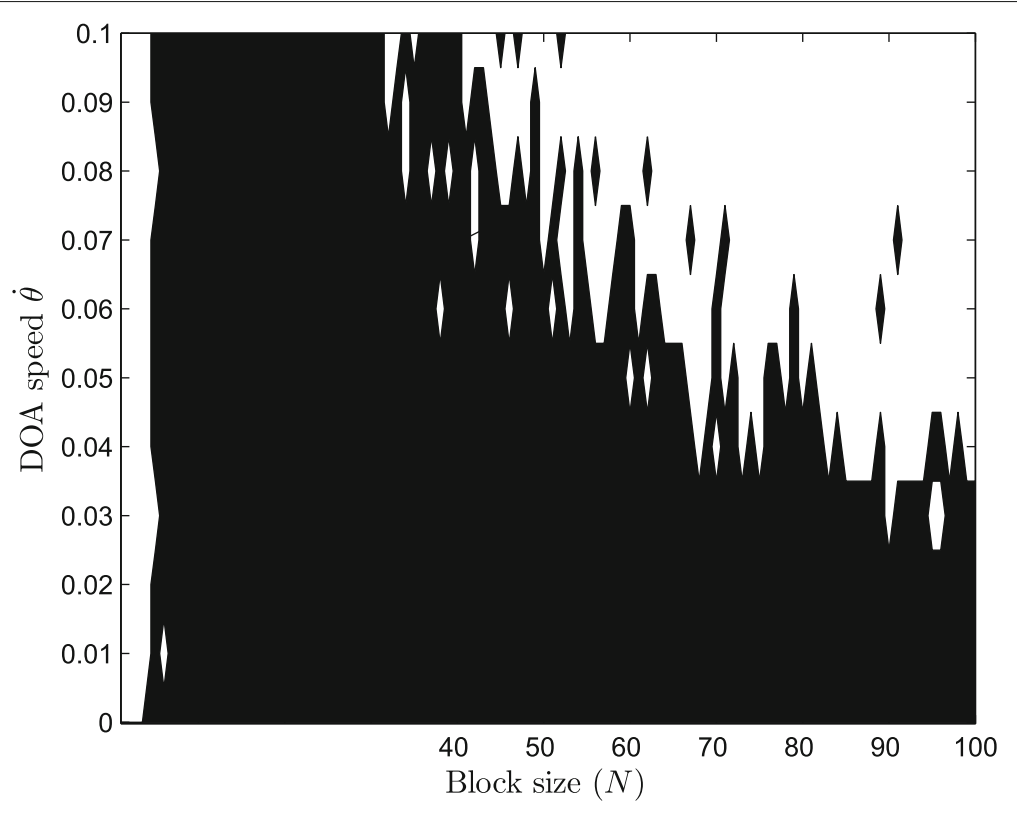

Fig. 7 Operational regions for the AF-based (white surface) and the root-MUSIC (black surface) estimators with $K=2, N_{a}=8$, and SNR $=25 \mathrm{~dB}$

argue that since the channel strengths are increasingly more accurate at higher SNR values, then the estimation error on the noise power should also remain constant and so does the SNR estimates. This is simply not true because as the true SNR increases, the true channel strength increases as well (for a fixed true noise variance) and the relative estimation error $\epsilon_{k}=\left|\hat{h}_{k}\right|^{2}-\left|h_{k}\right|^{2}$ is higher although the normalized error $\tilde{\epsilon}_{k}=\epsilon_{k} /\left|h_{k}\right|^{2}$ remains constant in average (i.e. the channel estimates' NRMSE remains constant). Consequently, larger $\left\{\epsilon_{k}\right\}_{k=1}^{K}$ yields a higher estimation error on the noise power (or equivalently the SNR); $\epsilon_{k}$ can be even larger than $2 \sigma^{2}$ to be estimated itself. For a larger number of receiving antenna elements ( $N_{a}=32$ for example), the SNR estimates are, however, reliable for the entire considered SNR region.

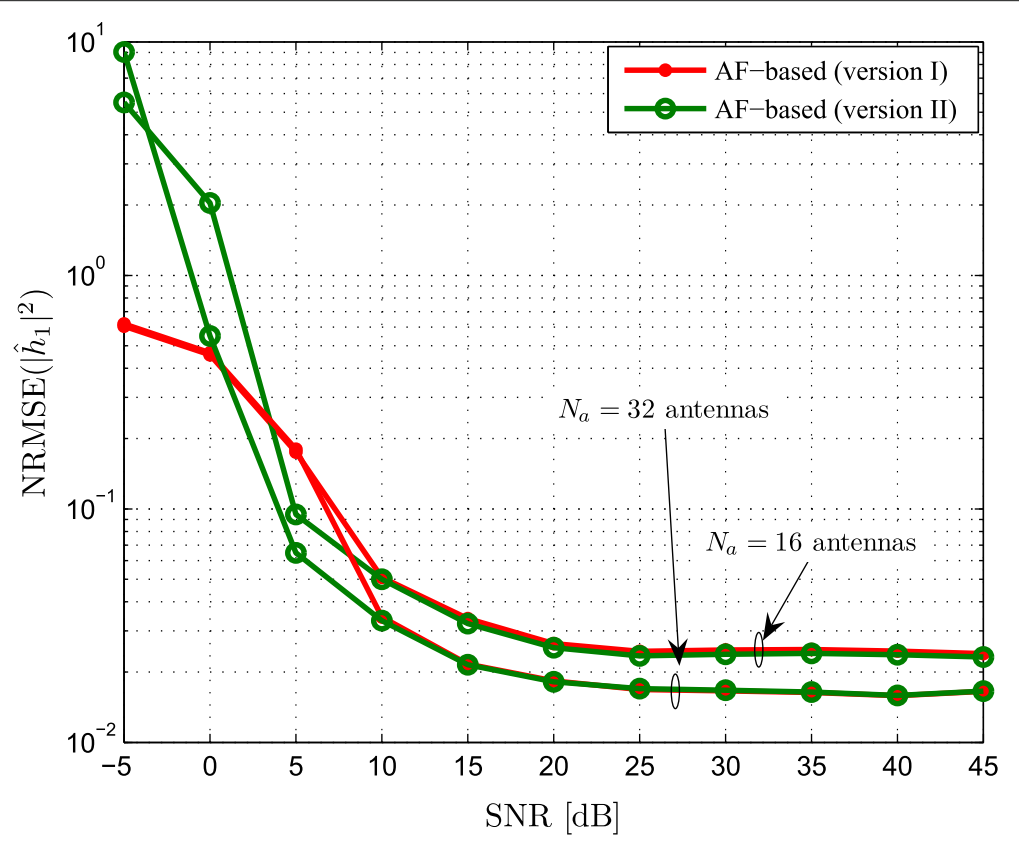

Fig. 8 NRMSE of the channel power estimators (versions I and II) with $N=10, \theta_{1}=18^{\circ}, \theta_{2}=36^{\circ}, K=2, N_{a}=16,32$, and $\sigma^{2}=2$ 


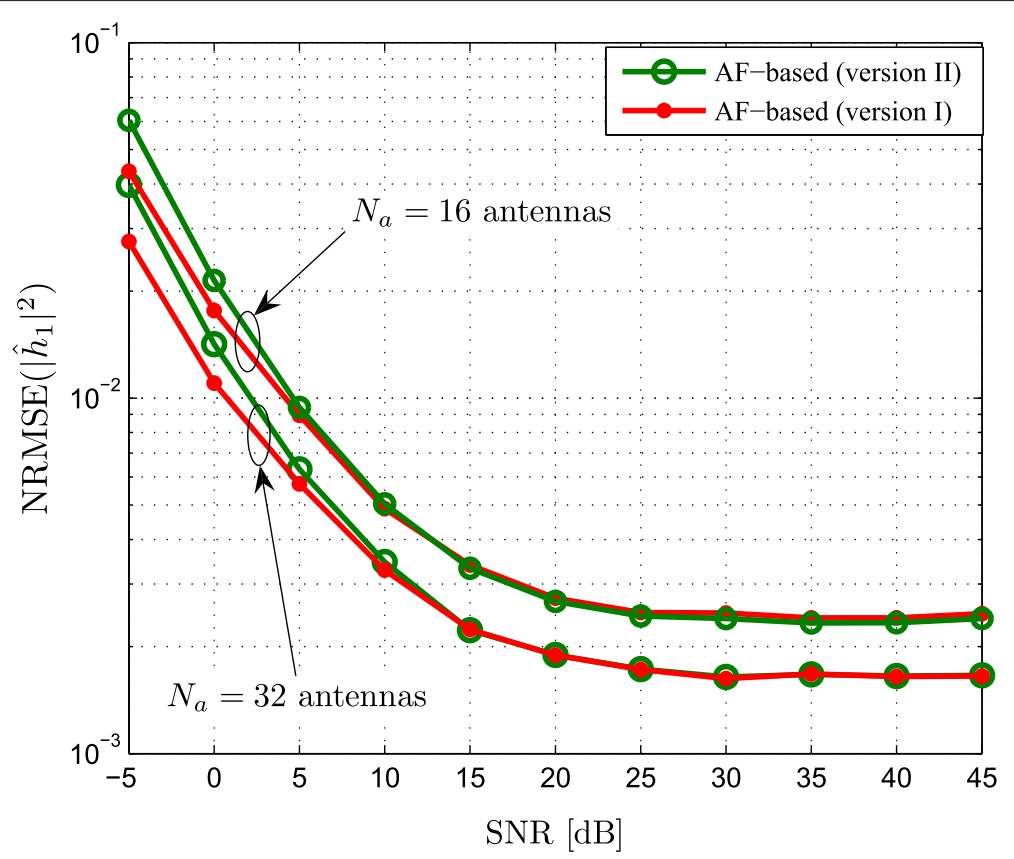

Fig. 9 NRMSE of the channel power estimators (versions / and II) with $N=1000, \theta_{1}=18^{\circ}, \theta_{2}=36^{\circ}, K=2, N_{a}=16,32$, and $\sigma^{2}=2$

\section{Conclusions}

In this paper, we derived a new DOA estimation method for multiple planar waves impinging on a ULA antenna array. The transmitted sources and the noise components are assumed to be spatially and temporally white. The new method is based on the annihilating filter technique. It was seen that the new method exhibits accurate statistical performance while having a low computational cost. Its major advantage is its capability of accurately resolving DOAs as close as $10^{\circ}$ from short data snapshots and even from a single snapshot. This capability makes this new estimator well geared toward applications that require

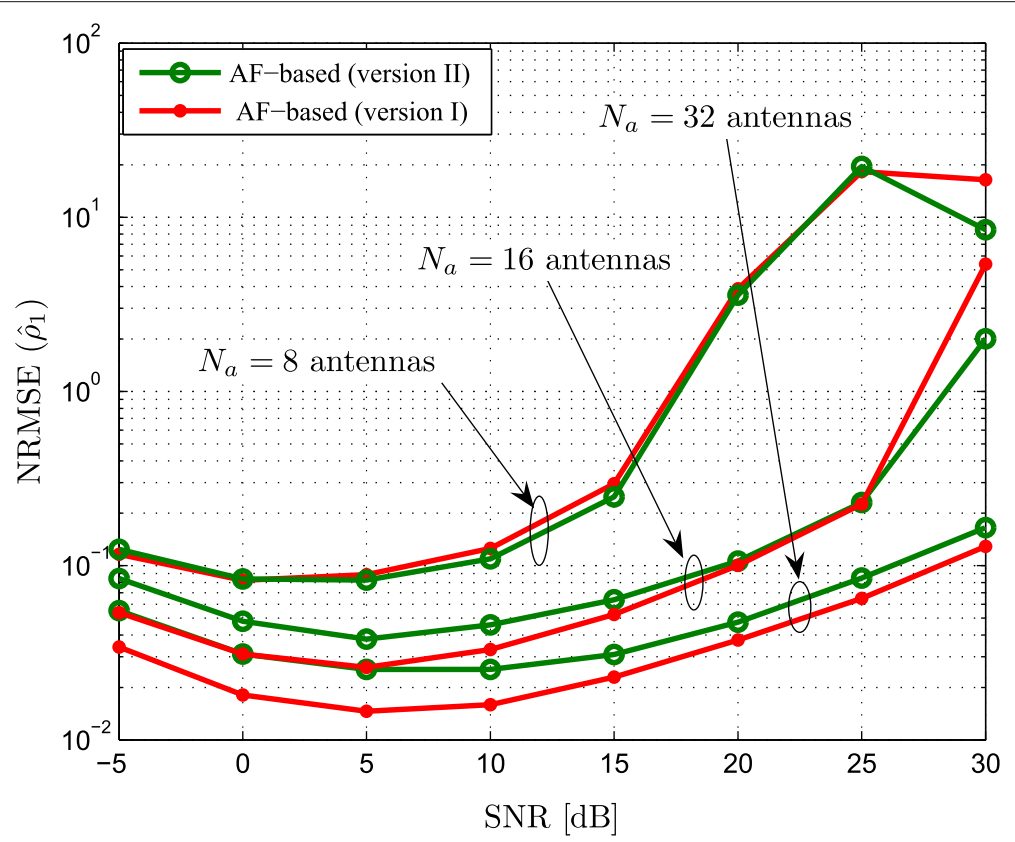

Fig. 10 NRMSE of the SNR estimators (versions / and II) with $\theta_{1}=18^{\circ}, \theta_{2}=36^{\circ}, K=2, N_{a}=8,16,32$, and $N=1000$ 
DOA estimation of fast moving sources or require up-todate estimates for the DOAs over very short observation windows. The estimated DOAs were then used to easily estimate the channel powers and SNRs for each source (or user).

\section{Endnotes}

${ }^{1}$ Extensions of the proposed AF-based technique to the problem of joint angle and delay estimation (JADE) [21] falls beyond the scope of this paper.

${ }^{2}$ The signal $a_{k}(n)$ can be complex symbols taken from any constellation such as QPSK, M-PSK and M-QAM or simply complex Gaussian.

${ }^{3}$ This is because all the cross-covariances that belong to any given secondary diagonal of the covariance matrix have the same expression.

${ }^{4}$ One could decide to consider the upper-triangular matrix, i.e. $i<l$. But this does not change the estimator, as seen from (5), since this will only introduce a negative sign in the exponential argument.

${ }^{5}$ Note that the vector $\boldsymbol{r}_{\theta}^{(l)}$ contains all the $N_{a}-l$ elements of the $l^{\text {th }}$ column that are lying under the main diagonal of the covariance matrix.

${ }^{6}$ We mention here that $\boldsymbol{r}_{\boldsymbol{\theta}}^{\prime(l)}$ plays the role of $\boldsymbol{r}_{\boldsymbol{\theta}}^{(l)}$ that was previously used when the estimation process was performed column-wise.

${ }^{7}$ This is because this version provides a larger number of estimates for each DOA, which can be averaged to obtain a more refined final estimate.

${ }^{8}$ Please note that the root-MUSIC techniques has almost the same complexity of the our AF-based estimator since it involves similar operations of SVD decomposition (but with different matrices sizes) and polynomial rooting. Also note that we evaluate and refer to the complexity of version I of the new AF-based estimator since it is more computationally expensive than version II.

${ }^{9}$ Their complexities also increase exponentially with the number of unknown DOAs, $K$, contrarily to the proposed estimator whose complexity increases only polynomially with $K$ (see Table 1 for $K=4$ ).

\section{Acknowledgments}

This work was made possible by NPRP grant NPRP 5-250-2-087 from the Qatar National Research Fund (a member of Qatar Foundation). The statements made herein are solely the responsibility of the authors. Work published in part in [22].

\section{Competing interests}

The authors declare that they have no competing interests.

\section{Publisher's Note}

Springer Nature remains neutral with regard to jurisdictional claims in published maps and institutional affiliations.

\section{Author details}

${ }^{1}$ INRS-EMT, 800, de la Gauchetière Ouest, Suite 6900, H5A 1 K6 Montréal, QC Canada. ${ }^{2}$ Texas A\&M University at Qatar, Engineering Building, Education City, Doha, Qatar.

Received: 2 November 2016 Accepted: 23 May 2017

Published online: 30 June 2017

\section{References}

1. DH Johnson, DE Dudgeon, Array Signal Processing Concepts and Techniques. (Prentice Hall, Englewood Cliffs, NJ, 1993)

2. HL Van Trees, Optimum Array Processing, 1st edn. (John Wiley, New York, 2002). Part IV of Detection, Estimation and Modulation Theory

3. TS Rappaport, Smart Antennas: Adaptive Arrays, Algorithms, and Wireless Position Location. (IEEE Press, New York, 1998)

4. SD Blostein, H Leib, Multiple antenna systems: role and impact in future wireless access. IEEE Commun. Mag. 41(7), 94-101 (2003)

5. A Jagannatham, B Rao, in Proc. of IEEE ACSSC'2006. Superimposed pilots vs. conventional pilots for channel estimation (IEEE, California, USA, 2011)

6. R Roy, A Paulraj, T Kailath, ESPRIT - A subspace rotation approach to estimation of parameters of cisoids in noise. IEEE Trans. Acoust. Speech Signal Proc. ASSP-34, 1340-1342 (1986)

7. P Stoica, KC Sharman, Maximum likelihood methods for direction-of-arrival estimation. IEEE Trans. Acoust. Speech Signal Proc. 38, 1132-1143 (1990)

8. M Agrawal, S Prasad, A modified likelihood function approach to DOA estimation in the presence of unknown spatially correlated Gaussian noise using a uniform linear array. IEEE Trans. Sign. Proc. 48(10), 2743-2749 (2000)

9. M Vetterli, P Marziliano, T Blu, Sampling signals with finite rate of innovation. IEEE Trans. Sign. Proc. 50(6), 1417-1428 (2002)

10. P Stoica, A Nehorai, Performance study of conditional and unconditional direction-of-arrival estimation. IEEE Trans. Acoust. Speech Signal Proc. 38(10), 1783-1795 (1990)

11. H Krim, M Viberg, Two decades of array signal processing research. IEEE Signal Proc. Mag. 13(4), 67-93 (1996)

12. BM Radich, KM Buckley, Single-snapshot DOA estimation and source number detection. IEEE Signal Proc. Lett. 4(4), 109-111 (1997)

13. RT O'Brien, K Kiriakidis, Single-snapshot robust direction finding. IEEE Trans. Signal Proc. 53(6), 1964-1978 (2005)

14. P Hacker, B Yang, Single snapshot DOA estimation. Adv. Radio Sci. 8 251-256 (2010). [Online]. Availablehttp://www.adv-radio-sci.net/8/251/ 2010/

15. S Moshavi, Multi-user detection for DS-CDMA communications. IEEE Commun. Mag. 34(10), 124-136 (1996)

16. ALC Hui, KB Letaief, Successive interference cancellation for multiuser asynchronous DS/CDMA detectors in multipath fading links. IEEE Trans. Commun. 46(3), 384-391 (1998)

17. JG Andrews, TH Meng, Optimum power control for successive interference cancellation with imperfect channel estimation. IEEE Trans. Wirel. Commun. 2(2), 375-383 (2003)

18. SP Weber, JG Andrews, X Yang, GD Veciana, Transmission capacity of wireless ad hoc networks with successive interference cancellation. IEEE Trans. Inf. Theory. 53(8), 2799-2814 (2007)

19. M Carlin, P Rocca, G Oliveri, F Viani, A Massa, Directions-of-arrival estimation through Bayesian compressive sensing strategies. IEEE Trans. Antennas Propag. 61(7), 3828-3838 (2013)

20. R Grover, DA Pados, MJ Medley, Subspace direction finding with an auxiliary-vector basis. IEEE Trans. Signal Proc. 55, 758-763 (2007)

21. JG Andrews, TH Meng, Optimum power control for successive interference cancellation with imperfect channel estimation. IEEE Trans. Wireless Commun. 2(2), 375-383 (2003)

22. SP Weber, JG Andrews, X Yang, GD Veciana, Transmission capacity of wireless ad hoc networks with successive interference cancellation. IEEE Trans. Inf. Theory. 53(8), 2799-2814 (2007) 